\title{
Dificuldades dos discentes na prática supervisionada de Fisioterapia na Unidade de
}

\section{Terapia Intensiva}

\author{
Difficulties of students in supervised Physiotherapy practice in an Intensive Care Unit \\ Dificultades de los estudiantes em la práctica supervisada de Fisioterapia em la Unidad de
} Cuidados Intensivos

Recebido: 01/10/2021 | Revisado: 09/10/2021 | Aceito: 13/10/2021 | Publicado: 16/10/2021

\author{
Luiz Euclides Coelho de Souza Filho \\ ORCID: https://orcid.org/0000-0002-0828-0911 \\ Universidade do Estado do Pará, Brasil \\ E-mail: luizcoelhodesouza@yahoo.com.br \\ Valéria Marques Ferreira Normando \\ ORCID: https://orcid.org/0000-0002-4234-5379 \\ Universidade do Estado do Pará, Brasil \\ E-mail: valeriafisio@gmail.com \\ Renato da Costa Teixeira \\ ORCID: https://orcid.org/0000-0002-4073-205X \\ Universidade do Estado do Pará, Brasil \\ E-mail: teixeirarenato@globo.com \\ Nathália Lima Costa \\ ORCID: https://orcid.org/0000-0002-1763-4785 \\ Universidade do Estado do Pará, Brasil \\ E-mail: nathaliaalc884@gmail.com \\ Angélica Menezes Bessa Oliveira \\ ORCID: https://orcid.org/0000-0001-7644-4538 \\ Universidade do Estado do Pará, Brasil \\ E-mail: angelbssa@ hotmail.com \\ Katiane da Costa Cunha \\ ORCID: https://orcid.org/0000-0002-5361-5090 \\ Universidade do Estado do Pará, Brasil \\ E-mail: Katianefisio@yahoo.com.br
}

\begin{abstract}
Resumo
Introdução: Sabe-se que, para a atuação ideal dos profissionais de saúde, sua formação deve ser realizada com qualidade. Por isso, a prática de estágios torna-se indispensável nas universidades, especialmente em áreas que demandam por muito conhecimento, como a Unidade de Terapia Intensiva (UTI). Assim, o objetivo desse estudo é analisar a percepção de estudantes de Fisioterapia sobre seus estágios supervisionados dentro de uma UTI. Métodos: Neste estudo transversal analítico e quantitativo, 29 alunos do $10^{\circ}$ semestre de Fisioterapia responderam a perguntas fechadas e abertas em que foram questionadas situações e experiências vivenciadas dentro do estágio na Unidade de Terapia Intensiva. Resultados e Discussão: Percebeu-se que a maioria dos alunos não haviam estado em unidades de terapia anteriormente, além de acharem o tempo de estágio inadequado. Contudo, grande parte dos alunos sentem-se aptos para o trabalho nesse setor e acreditam que o estágio foi um grande aprendizado para melhoria da prática clínica e da humanização do paciente. Conclusão: Os alunos de fisioterapia ainda possuem dificuldades, enquanto realizam os estágios, que podem ser sanadas com maior engajamento da coordenação de seus cursos e de seus professores. Apesar disso, a oportunidade de estagiar em Unidades de Terapia é fundamental para o crescimento profissional destes estudantes.
\end{abstract}

Palavras-chave: Educação superior; Estágio; Hospitais de ensino.

\begin{abstract}
Introduction: For the ideal performance of health professionals, their training must be carried out with quality. Therefore, the practice of internships becomes indispensable in universities, especially in areas that demand a lot of knowledge, such as the Intensity Therapy Unit (ICU). Thus, the objective of this study is to analyze the perception of Physiotherapy students about their supervised internships in an ICU. Methods: In this cross-sectional analytical and quantitative study, 29 students from the 10th semester of Physiotherapy answered closed and open questions in which situations and experiences during the internship in the Intensive Care Unit. Results and Discussion: Most students had not been in therapy units previously, besides finding the internship time inadequate. However, they feel able to work in this sector and believe that the internship was a great learning experience for improving clinical practice and
\end{abstract}


humanizing the patient. Conclusion: Physiotherapy students still have difficulties, while doing internships, which can be remedied with greater engagement in the pedagogical coordination of their courses and their teachers. However, the opportunity to intern in Therapy Units is fundamental for the professional growth of these students.

Keywords: Education, higher; Internships; Hospitals, teaching.

\section{Resumen}

Introducción: Se sabe que, para el óptimo desempeño de los profesionales de la salud, su formación debe realizarse con calidad. Por lo tanto, la práctica de las pasantías se vuelve indispensable en las universidades, especialmente en áreas que demandan mucho conocimiento, como la Unidad de Cuidados Intensivos (UCI). Así, el objetivo de este estudio es analizar la percepción de los estudiantes de Fisioterapia sobre sus prácticas supervisadas dentro de una UCI. Métodos: En este estudio transversal analítico y cuantitativo, 29 estudiantes del décimo semestre de Fisioterapia respondieron preguntas cerradas y abiertas en las que se cuestionaron situaciones y experiencias vividas dentro del internado en la Unidad de Cuidados Intensivos. Resultados y Discusión: Se notó que la mayoría de los estudiantes no habían estado antes en unidades de terapia, además de encontrar el tiempo de pasantía inadecuado. Sin embargo, la mayoría de los estudiantes se sienten capaces de trabajar en este sector y creen que la pasantía fue una gran experiencia de aprendizaje para mejorar la práctica clínica y la humanización del paciente. Conclusión: Los estudiantes de fisioterapia siguen teniendo dificultades durante las prácticas, que pueden remediarse con un mayor compromiso mediante la coordinación de sus cursos y sus profesores. A pesar de esto, la oportunidad de realizar prácticas en Unidades de Terapia es fundamental para el crecimiento profesional de estos estudiantes.

Palabra clave: Educación superior; Prácticas; Hospitales docentes.

\section{Introdução}

A Fisioterapia tornou-se uma graduação de ensino superior regulada no Brasil em 1969. Com o passar dos anos, a profissão cresceu e o profissional fisioterapeuta adquiriu responsabilidades que vão além de somente reabilitar o paciente (Simoni, 2015). Atualmente, as faculdades de Fisioterapia possuem por objetivo a formação de profissionais humanizados, capacitados para atuarem em equipes multiprofissionais, que consigam promover atenção integral ao paciente (Andrade, 2010). Para tanto, é indispensável que o futuro fisioterapeuta possua estágio supervisionado durante a graduação, buscando complementar sua formação educacional a partir de experiências reais que não apenas são educativas, mas que também iniciam o aluno na prestação de serviço para comunidade (Viana et al., 2012).

Nesse contexto, percebe-se que a formação de profissionais da saúde está atrelada a uma educação clínica de qualidade. Esta educação, por sua vez, depende diretamente da prática clínica oferecida pelos estágios, que devem ser realizados em hospitais, com pacientes reais e supervisionada por professores capacitados. Essa dinâmica de estágios é necessária para que o aluno possa aplicar o conhecimento que já possui e também para que o aluno possa compreender quais dificuldades da prática ainda devem ser sanadas antes de sua formação (Aljadi et al., 2017).

Dentre essas atividades práticas fornecidas pelos estágios, está a prática na Unidade de Terapia Intensiva (UTI). O estágio na UTI causa ansiedade nos alunos de fisioterapia não apenas porque, muito em breve, eles poderão fazer parte da equipe multiprofissional de terapias intensivas, mas também porque irão lidar com atividades mais complexas e com situações mais críticas, que exigem maior preparo técnico e emocional do aluno e de seu preceptor (Motter et al., 2014).

Contudo, a realização da prática de estágio vem sendo conturbada nos últimos anos. No Brasil, percebeu-se um aumento no número de cursos de Fisioterapia, que atualmente já somam 500, sendo mais de $80 \%$ ofertados por escolas privadas (Góes et al., 2017). Esse cenário, somado à dificuldade na liberação de recursos na qual as faculdades públicas estão inseridas, fazem com que os estágios supervisionados não estejam acontecendo de maneira adequada, deixando lacunas na formação dos futuros fisioterapeutas que estrarão no mercado de trabalho.

Por isso, esse trabalho possui como objetivo analisar qual a percepção dos acadêmicos de Fisioterapia sobre seus estágios supervisionados, visando reconhecer quais as dificuldades enfrentadas pelos alunos durante atividade prática na UTI. 


\section{Metodologia}

Trata-se de um estudo transversal analítico e quantitativo realizado em julho de 2019, no município de Belém, com estudantes do $10^{\circ}$ semestre do curso de Fisioterapia da Universidade do Estado do Pará que cumpriram estágio supervisionado curricular nos hospitais vinculados ao seu Projeto Político Pedagógico do Curso (PPC). Para participar da pesquisa, todos os indivíduos selecionados tiveram que assinar o Termo de Consentimento Livre e Esclarecido (TCLE). O estudo foi aprovado pelo Comitê de Ética em Pesquisa da Universidade do Estado do Pará sob parecer 3.423.127 e seguiu os princípios e diretrizes da resolução 466/12 do Conselho Nacional de Pesquisa em Saúde que envolve seres humanos e a Declaração de Helsinque e o Código Nuremberg. Não houve identificação dos participantes individualmente, respeitando assim a privacidade destes.

Todo os estudantes de fisioterapia do $10^{\circ}$ semestre foram incluídos na pesquisa, totalizando 29 discentes. Utilizou-se um questionário contendo dez perguntas relativas a aspectos sobre a experiência da prática supervisionada em Terapia Intensiva. Seis perguntas possuíam respostas seguindo a escala Linkert, que foram codificadas para análise de dados em números crescentes, sendo 1 para discordo totalmente e 5 para concordo totalmente. Os alunos foram indagados sobre seus conhecimentos prévios acerca das habilidades exigidas no atendimento fisioterapêutico na UTI; sobre a adequação do tempo de prática dos estágios na UTI, de 12 dias e 5 horas diárias, proporcionado pela Universidade; foram questionados sobre o tamanho dos grupos em que a turma era subdividida. Somado a isso, os participantes da pesquisa também responderam perguntas acerca de suas melhorias na prática clínica após o estágio e se estavam preparados para trabalhar em UTI. Como última pergunta com respostas baseada na escala Likert, foi interrogado aos alunos se eles sentiram apoio da equipe multiprofissional de UTI do ambiente em que estagiaram. Além disso, foram feitas duas perguntas com respostas de “sim” ou "não" sobre as impressões iniciais dos estudantes acerca da prática do estágio: 1) Houve feedback acerca da sua prática por parte do professor/preceptor? e 2) Você já havia estado em uma UTI antes do estágio curricular?”. Por último, os participantes responderam as seguintes perguntas abertas: “Quais sensações/sentimentos que você considerou positivo no seu contato na prática supervisionada em UTI?” e "Para você, qual ou quais foram as principais barreiras na prática supervisionada em UTI?". Os dados foram organizados e apresentados em gráficos do Microsoft Excel e os alunos foram identificados com a letra E e um número de 1 a 29, de acordo com a ordem alfabética de seus nomes.

\section{Resultados}

Foi gerada uma amostra de 29 estudantes, composta por 22 mulheres e 7 homens. Os participantes estavam no $10^{\circ}$ semestre do curso de Fisioterapia e haviam completado seus estágios supervisionados em Unidades de Terapia Intensiva (UTI) em 3 hospitais de Belém.

A Figura 1 mostra sobre as impressões iniciais do discente no ambiente da terapia intensiva. Nela observou-se que $72,5 \%(21 / 29)$ nunca estiveram nesse local e que 90,0\% (26/29) receberam feedback positivo por parte do professor/preceptor. 
Research, Society and Development, v. 10, n. 13, e371101321323, 2021

(CC BY 4.0) | ISSN 2525-3409 | DOI: http://dx.doi.org/10.33448/rsd-v10i13.21323

Figura 1 - Impressões iniciais sobre a prática supervisionada. (n=29).

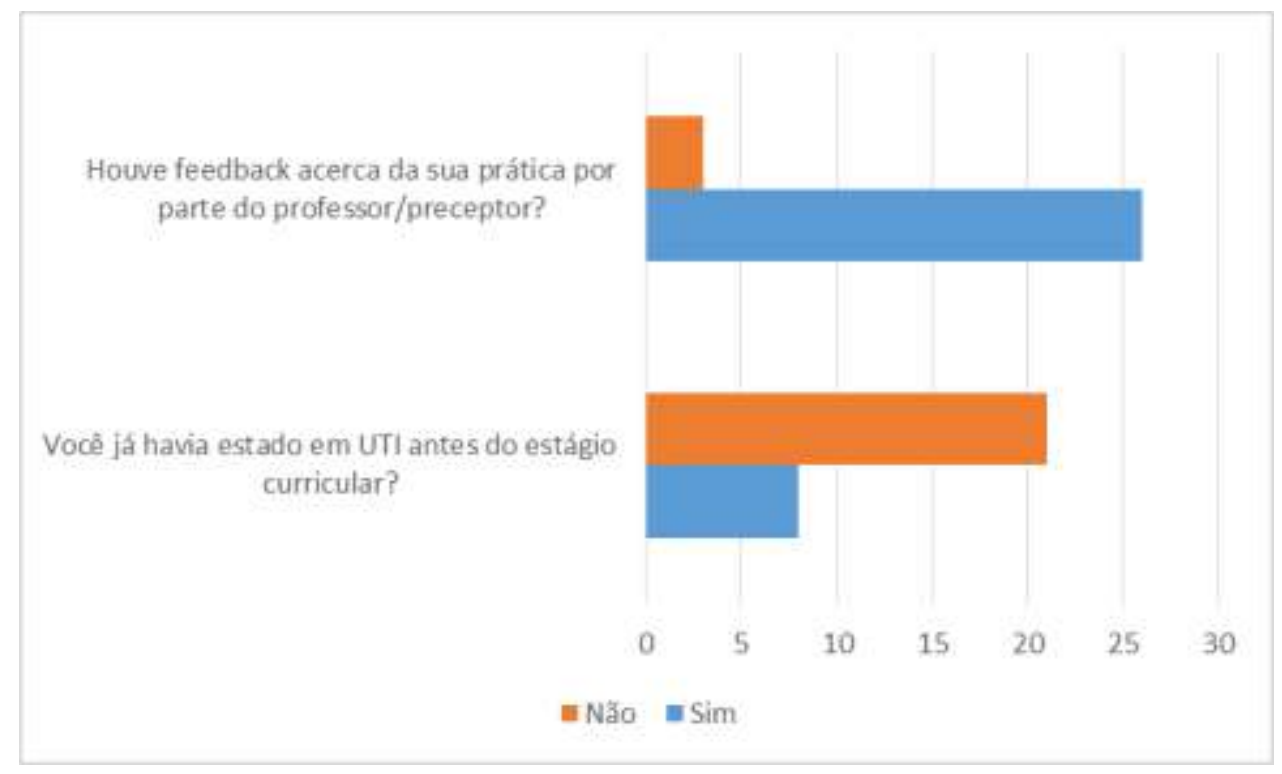

Fonte: Autores.

As perguntas subsequentes buscaram analisar a relação dos estudantes com a UTI. Os resultados destas 6 perguntas estão presentes na Figura 2.

Figura 2 - Perguntas com respostas baseadas na escala Linkert. $(\mathrm{n}=29)$.

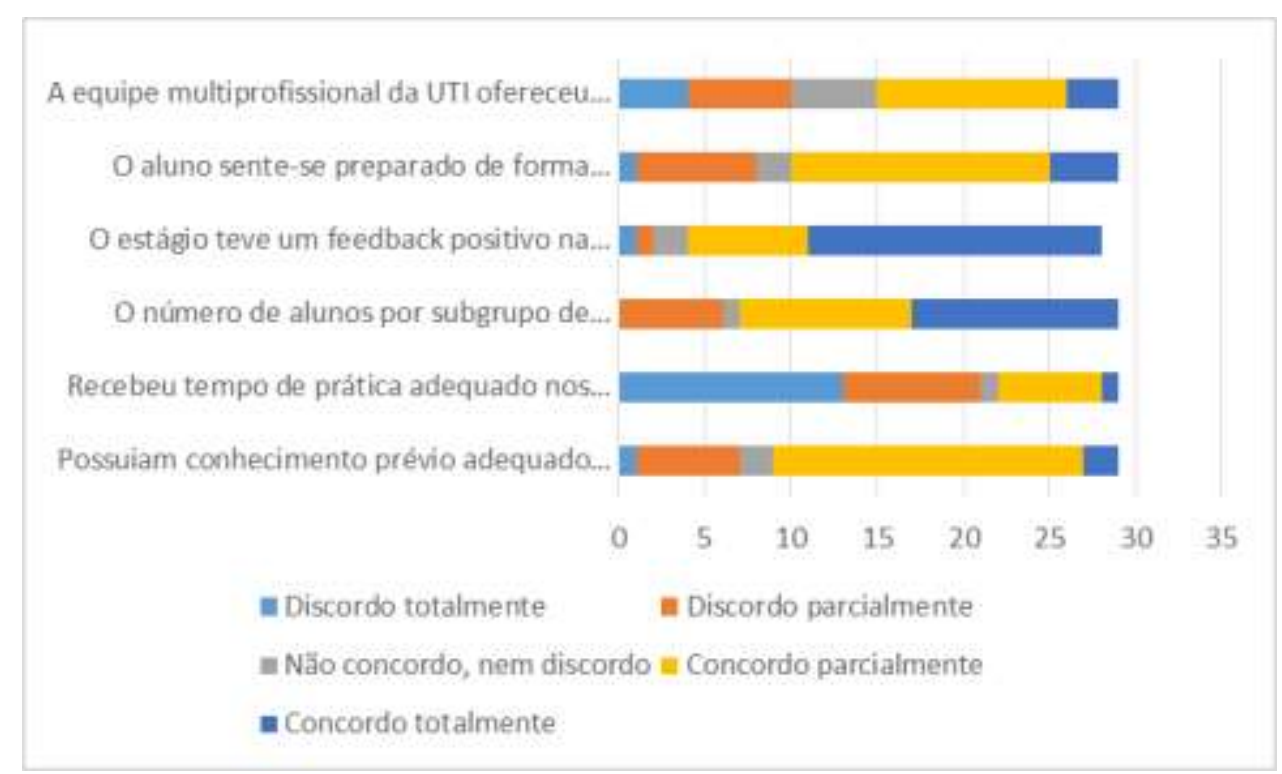

Fonte: Autores.

Entre as perguntas listadas na Figura 2, inicialmente foi perguntado se a equipe multiprofissional da UTI ofereceu apoio aos estudantes e 11 responderam "concordo parcialmente". Também foi questionado se o aluno se sente preparado de forma adequada para exercer seu trabalho na UTI, onde a maioria (15/29) também responderam "concordo parcialmente".

Além disso, questionou-se “O estágio teve um feedback positivo para a sua melhoria no cenário de prática?”, com resposta a positiva de "concordo totalmente" para 17 alunos. Somado a isso, os alunos foram interrogados acerca do número de 
alunos por subgrupo durante o estágio, o que foi bem avaliado pelos participantes, visto que 12 responderam "concordo totalmente" e 10 responderam "concordo parcialmente".

Nas duas últimas perguntas expostas na Figura 2, perguntou-se se os alunos achavam o tempo de estágio adequado, sendo esta a pergunta com maior índice de respostas negativas, pois 13 alunos deram como resposta "discordo totalmente" e 8 responderam "discordo parcialmente". Finalizou-se, então, perguntando se os participantes acreditavam possuir conhecimento prévio adequado para as habilidades prévias exigidas no atendimento fisioterapêutico na UTI e 18 afirmaram "concordo parcialmente".

Por último, os alunos responderam perguntas abertas. A primeira pergunta deste tipo foi "Quais sensações/sentimentos que você considerou positivo no seu contato na prática supervisionada em UTI?" e as respostas em grande parte foram relacionadas ao cuidado com o paciente, citando-se palavras como "atenção", "empatia", "ajuda", "humanização". A segunda pergunta aberta foi "Para você, qual ou quais foram as principais barreiras na prática supervisionada em UTI?”. A principal barreira observada foi em relação ao tempo do estágio, sendo uma crítica comum no relato de 11 alunos.

"O tempo de atendimento, pois quase sempre outros profissionais queriam atender o paciente no momento em que se daria início a fisioterapia. Impossibilitando o tratamento adequado devido ao curto período de tempo.” (E28)

Em outros trechos, 8 respostas citaram como barreira a falta de acolhimento dos profissionais de outras áreas com os alunos, com fragilidade na integração da equipe multiprofissional.

"[...] falta de apoio e incentivo interdisciplinar, equipe individualista e não tem momentos de conversa interdisciplinar." (E6)

“[...] senti a falta da relação da equipe multiprofissional” (E2)

Uma outra barreira encontrada, em 6 respostas, foi quanto ao papel do professor/preceptor durante o estágio. Em alguns relatos percebeu-se reclamações quanto a falta participação, amparo e diálogos do professor com os alunos.

"Pouco tempo de prática, poucas habilidades adquiridas e pouco diálogo teórico com o Professor." (E8)

“[...] falta de amparo pelo professor.” (E19)

\section{Discussão}

O presente estudo buscou analisar a percepção de estudantes do último ano de Fisioterapia acerca de seus estágios supervisionados em Unidades de Terapia Intensiva. Os estágios durante a graduação além de serem preconizados pela base curricular dos cursos, são fundamentais no sentido de desenvolverem competências e habilidades. Os graduandos são estimulados a atuarem individualmente e em equipe, ao praticarem a interdisciplinaridade. Além disso, desenvolvem a habilidade de comunicação, de liderança e de gestão dentro dos hospitais, imprescindíveis na atuação profissional independentemente do nível de atenção em saúde que virão a trabalhar (Lima, 2020). Essas características que devem vir com os discentes por vezes não é parte deste no início do estágio cabendo ao formador da área de estágio detectar possíveis lacunas dos estudantes e adequar o estágio visando uma melhor adaptação estágio/discente (Filho et al., 2021). 
Na composição das equipes de UTI, é possível encontrar médicos, fisioterapeutas, enfermeiros, psicológicos, assistentes sociais, nutricionistas. Dentro desse contexto, o trabalho multiprofissional vem em busca de humanizar o atendimento dos pacientes em estado crítico e de somar opiniões para um tratamento adequado, sendo um profissional passível de complementar a conduta de outro (Evangelista et al., 2016). Assim, fica evidente a necessidade do respeito e do acolhimento entre os profissionais de saúde no trabalho com pacientes de alto risco. Nesse sentido, percebeu-se as divergências de opiniões entre os alunos no que diz respeito ao sentimento de acolhimento por parte da equipe multiprofissional com que estagiaram, pois a resposta mais assinalada, "concordo parcialmente", foi comum em apenas 37,93\% dos alunos. Somado a isso, o trabalho da equipe profissional foi questionado no relato de alguns participantes. Relatou-se a falta de trabalho integral, percebendo-se certa desorganização com o tempo de atendimento adequado entre os diferentes profissionais, além de pouca receptividade com os alunos, que ainda estavam em processo de aprendizagem. Logo, percebe-se que na prática, o atendimento não vem ocorrendo de forma integrada e multiprofissional no cuidado progressivo ao paciente crítico ou grave, conforme preconiza o Ministério da Saúde (2017), o que pode gerar comportamentos iatrogênicos e pode afetar a qualidade de aprendizagem dos profissionais em formação.

Quanto ao papel do fisioterapeuta na UTI, sabe-se que a sua atuação está tanto no momento de atendimento a pacientes admitidos em estado crítico, como também na promoção de assistência aos pacientes que receberam alta da UTI, visando evitar complicações respiratórias, cardíacas e musculares principalmente (Motter et al., 2014) Logo, o estágio é fundamental para que os estudantes compreendam quais são suas responsabilidades e como lidar com elas sob pressão. Neste estudo, 65,5\% dos participantes afirmam concordar, parcialmente ou totalmente, com a questão de se sentirem aptos para trabalhar adequadamente na terapia intensiva. Esse resultado vai ao encontro com o estudo de Gaiad e Sant'Ana, 2005, realizado com egressos de Fisioterapia, onde $85,7 \%$ dos participantes sentiram-se aptos para atuarem como fisioterapeutas a partir da prática desenvolvida nos estágios supervisionados. Contudo, o fisioterapeuta, apesar de ser parte integrante do corpo técnico ainda busca seu espaço junto às profissões mais enraizadas no âmbito da UTI, principalmente quando visto em centros mais afastados das metrópoles. Prova disso pode ser visto em estudos atuais que não consideram o profissional fisioterapeuta como formador da equipe base (Brasil, 2010; Holstein, Liano, Castro, 2017; Silva et al., 2019).

Os estágios também foram fundamentais para construção da prática clínica e criação de uma relação positiva com o paciente. Nos relatos dos estudantes, foi possível compreender que o atendimento em UTI desenvolveu o sentimento de empatia pelos pacientes, trazendo uma sensação atendimento de segurança na execução de atendimentos considerados humanizados. "Aprendizado e autoconfiança.", "Humanização e tranquilidade.", "Sentimento de empatia pelos pacientes, de melhora do ato do cuidado e de melhora do raciocínio clínico durante as avaliações diárias." foram algumas das respostas obtidas no que diz respeito a sensações e sentimentos considerados positivos na prática em UTI. Tais relatos são interessantes pois demonstram que os alunos compreendem a necessidade de um maior cuidado e maior atenção para os doentes de UTI, mas também sabem que é um ambiente hospitalar carente de esperanças, onde um atendimento humanizado é fundamental parar gerar mudanças tanto no paciente, quanto em seus familiares (Bezerra, Passos, 2020). Visões similares são vistas em outras graduações como da enfermagem em que o processo de cuidar e a proximidade paciente/profissional é tão equânime quanto ao profissional fisioterapeuta. Tais resultados corroboram com a formação desses futuros profissionais e a visão das Diretrizes Curriculares Nacionais quanto ao perfil buscado para esse futuro egresso (Brasil, 2002; Castro et al., 2019; Gomes, Souza, Araújo, 2020)

Apesar da sensação de aptidão profissional, dentre as reclamações dos alunos e como a questão mais mal avaliada, tem-se o tempo dos estágios. No programa curricular da universidade dos alunos participantes, preconiza-se 24 horas semanais de estágio em UTI durante 7 semanas. Contudo, participantes relataram que os estágios costumavam ocorrer em 12 dias, com 5 horas semanais. Por isso, 72,4\% dos alunos discordaram, parcialmente ou totalmente, quanto à adequação do tempo dos 
estágios. Este ponto também condiz com o encontrado em outros estudos. Na pesquisa de Gaiad e Sant'Ana (Gaiad, Santïana, 2005), 57,1\% dos participantes consideraram a carga horária do seus estágios insuficientes. Já no estudo de Viana et. al (2012), $57,4 \%$ dos estudantes de fisioterapia possuíam estágios que aconteciam de uma a três vezes na semana. Dessa forma, ao analisar estudos realizados em diferentes anos, fica evidente que os discentes de Fisioterapia da atualidade ainda estão se deparando com dificuldades vivenciadas por discentes do início do século XXI. Tal resultado, demonstra uma falha na prática da quantidade de cargas horarias preconizadas pelas faculdades, apontando para a necessidade de reestruturação da matriz curricular dos cursos de graduação em Fisioterapia.

O estágio curricular supervisionado é o elo entre os futuros formandos e os seus futuros locais de trabalho, sendo esse momento fundamental para a formação destes não somente nos aspectos procedimentais como também nos aspectos filosófico, humano e comportamental destes. Não obstante, as leis e os regimentos que norteiam o estágio supervisionado devem ser entendidos por todos que compõem esse pilar acadêmico, pois o bom entendimento destes pode agregar a evolução do estágio, tendo com o olhar das novas Diretrizes Curriculares e o papel articulado das metodologias ativas todos, discentes e docentes, uma integralização dos direitos, deveres e prática nos locais de estágio (Carvalho, Souza, 2020; Ferreira, Rocha, 2020; Pereira, Nunes, 2020).

Um outro ponto que diz respeito aos cursos de graduação, é a atitude dos preceptores. Estes, na opinião dos participantes, foram considerados obstáculos para prática adequada durante o estágio. "Tempo de prática [...] que poderia ser prejudicado por interferências pessoais do docente.", "Falta de supervisão adequada por parte do professor." foram algumas das situações apontadas como barreira para prática. No estudo de Aljadi et. al (2017), analisou-se as dificuldades na prática dos estágios para os preceptores. Como desafio, pagamentos atrasados e salários baixos foram fatores identificados como desestimulantes. Somado a isso, alguns preceptores apontam como problema a chegada de alunos sem bases de habilidades práticas e de conhecimentos teóricos, o que atrasa os atendimentos e gera dificuldades no gerenciamento de tempo (Aljadi $e t$ al., 2017; Lacerda et al., 2019). Outro fator importante é a formação profissional desses preceptores que muitas vezes não possuem um perfil profissional adequado para a realização da atividade com excelência (Alves et al., 2020). Dessa forma, é nítida a falta de diálogo entre os professores e a coordenação dos cursos, de forma a demonstrem as dificuldades enfrentadas por eles e pelos futuros egressos.

\section{Conclusão}

Os alunos participantes da pesquisa relataram que o estágio supervisionado foi benéfico para as suas práticas profissionais, de modo que eles não somente se sentem preparados para atuar na equipe multiprofissional de uma UTI, mas que também observaram a possibilidades de colocar em prática seus conhecimentos teóricos de clínica atrelado a um atendimento baseado na empatia e na humanização. Contudo, as universidades, na figura das coordenações do curso de Fisioterapia e dos professores, ainda podem melhorar a organização dos estágios e os profissionais de hospitais escolas podem ser mais receptivos aos alunos. É válido salientar que esse estudo possui como limitação a pequena amostra de participantes. Logo, estudos com amostras maiores, incluindo alunos de outros semestres e de diferentes faculdades, seriam benéficos na validação dos resultados apresentados.

\section{Referências}

Aljadi, S. H., Alotaibi, N. M., Alrowayeh, H. N., \& Alshatti, T. A. (2017). Benefits and Challenges of Supervising Physical Therapy Students in the State of Kuwait: A National Study. Journal of allied health, 46(4), 243-249.

Alves, F. A. D., de Oliveira, B. C., dos Santos, F. C., do Nascimento Matta, R. E., Silva, H. G. N., de Sousa, C., \& de Carvalho, A. F. M. (2020). Perfil dos fisioterapeutas nas unidades de terapia intensiva adulto. Revista Eletrônica Acervo Saúde, (55), e4068-e4068. 
Andrade, P. M. D. O. (2010). Avaliação das diretrizes curriculares conforme a perspectiva biopsicossocial da organização mundial de saúde. Avaliação: Revista da Avaliação da Educação Superior (Campinas), 15, 121-130.

Brasil. Conselho Nacional de Educação. Câmara de Educação Superior Resolução n. 4 de 19 de fevereiro de 2002. Institui as Diretrizes Curriculares Nacionais do curso de graduação em Fisioterapia. 2002. http://portal.mec. gov.br/cne/arquivos/pdf/CES 042002.pdf

Brasil, Ministério da Saúde - ANVISA - Resolução-RDC nº 7, de 24 de fevereiro de 2010, http://www.amib.org.br/pdf/RDC-07-2010.pdf.

de Carvalho Coelho, C. L., de Azevedo, I. F., \& de Sousa, R. T. (2020). A disciplina Estágio Supervisionado na perspectiva de uma formação integral. Research, Society and Development, 9(8), e620985252-e620985252.

da Silva Bezerra, N. K. M., \& dos Passos, D. F. (2020). Inserção De Graduandos Em Fisioterapia Na Uti: Implicações Para A Formação Integral. Revista de Educação da Universidade Federal do Vale do São Francisco, 10(23), 110-127.

da Silva Castro, A., Arboit, É. L., Ely, G. Z., Dias, C. A. M., Arboit, J., \& Camponogara, S. (2019). Percepções da equipe de enfermagem acerca da humanização em terapia intensiva. Revista Brasileira em Promoção da Saúde, 32.

de Lacerda, L. C. A., Lima, N. X., Ribeiro, D. R., \& de Omena Messias, C. M. B. (2019). Percepção do graduando em saúde sobre preceptoria e prática interdisciplinar em ambiente hospitalar. Ciências \& Cognição, 24(1).

de Lima, E. S. (2020). Potencialidades e desafios de um estágio curricular obrigatório em Fisioterapia: percepções de um estagiário. Revista Docência do Ensino Superior, 10, 1-18.

de Souza Filho, L. E. C., da Costa Cunha, K., de Souza Santos, M. C., Falcão, L. F. M., \& Normando, V. M. F. (2021). Impacto de um vídeo educativo de sistematização para exame físico na prática discente de fisioterapia em Unidade de Terapia Intensiva. Research, Society and Development, 10(5), e26910514940-e26910514940.

de Souza Soares ${ }^{1}$, A. L., Conceiçãoํㅗ T. P., \& Monteiro, V. R. (2019). A Integração Da Equipe Multiprofissional Na Unidade De Terapia Intensiva: Revisão Sistemática. Revista CPAQV_Centro de Pesquisas Avançadas em Qualidade de Vida| Vol, 11(3), 2.

Evangelista, V. C., Domingos, T. D. S., Siqueira, F. P. C., \& Braga, E. M. (2016). Equipe multiprofissional de terapia intensiva: humanização e fragmentação do processo de trabalho. Revista Brasileira de Enfermagem, 69, 1099-1107.

Ferreira, R. K. R., \& Rocha, M. B. (2020). A importância das práticas educativas do estágio supervisionado na formação do enfermeiro: uma revisão integrativa. Research, Society and Development, 9(4), e121942933-e121942933.

Gaiad, T. P., \& Sant'Ana, D. D. M. G. (2005). Análise da eficácia do estágio supervisionado em fisioterapia na formação profissional: uma visão do egresso. Arquivos de Ciências da Saúde da UNIPAR, 9(2).

Góes, A. B. D., Araújo, F. R. O. D., Marques, A. P., \& Schmitt, A. C. B. (2017). Overview of physical therapy graduation courses in Brazil: current scenario. Fisioterapia em Movimento, 30, 661-669.

Gomes, A. P. R. S., Souza, V. C., \& de Oliveira Araujo, M. (2020). Atuação do enfermeiro no cuidado humanizado em unidades de terapia intensiva no Brasil: uma revisão integrativa da literatura. HU Revista, 46, 1-7.

Holstein, J., Liano, M. S., \& de Castro, A. A. M. (2017). Inserção Do Fisioterapeuta Em Equipe Multiprofissional, Nos Serviços De Urgência E Emergência: Relato De Experiência. Anais do Salão Internacional de Ensino, Pesquisa e Extensão, 9(1).

Ministério Da Saúde. Portaria No 2.436Brasília, 2017. <https://bvsms.saude.gov.br/bvs/saudelegis/gm/2017/prt243 6_22_09_2017.html>.

Motter, A. A., Vieira, L. A., Bertola, I. P., \& Ferreira, M. P. (2014). Sentimentos vivenciados por acadêmicos de Fisioterapia ao estagiar em unidade de terapia intensiva. Cad Edu Saude e Fis, 1(2), 73-84.

Pereira, M. D., Pereira, M. D., \& Nunes, A. K. F. (2020). Estágio curricular supervisionado em Psicologia Clínica à luz das DCNs. Research, Society and Development, 9(8), e440985900-e440985900.

Simoni, D. E., Carvalho, J. B., Moreira, A. R., Morera, J. A. C., Maia, A. R. C., \& Boreinstein, M. S. (2015). A formação educacional em fisioterapia no Brasil: fragmentos históricos e perspectivas atuais. Hist enferm Rev eletronica, 6(1).

Viana, R. T., Moreira, G. M., Melo, L. T. M., De Sousa, N. P., Brasil, A. C. D. O., \& Abdon, A. P. V. (2012). O estágio extracurricular na formação profissional: a opinião dos estudantes de fisioterapia. Fisioterapia e Pesquisa, 19, 339-344. 